\title{
Design of Intelligent Parking System Based on Cloud Platform
}

\author{
Juan Fang, Ze-Qing Chang, Yan-Jin Cheng, Min Cai, Ju Li, Zeng-Jun Chen \\ College of Computer Science, Beijing University of Technology \\ Beijing, Chinaa \\ Email: fangjuan@bjut.edu.cn
}

\begin{abstract}
With the increasing number of private cars in urban areas year by year, the problems such as parking difficulties and traffic congestion become the new trend of urban development, the informationization is supposed as an important means to solve the problem of urban parking. It is researched on urban parking management solution in the Background of Big Data, and the Big Data technologies is utilized to effectively integrate, analyze and forecast traffic conditions, parking data and user behavior, and upgrade the traditional parking industry to intelligent parking. Developed with android platform, the function of user location, parking lot preselection, driving route planning, parking lot details and parking charge are deeply analyzed and designed, which realizes intelligent parking real-time query system based on Android, to help the majority of car owners access to parking information in real-time to solve the "parking difficult" problem.
\end{abstract}

Keywords-Intelligent parking ; Cloud platform; Performance.

The parking difficulties is the static traffic problem which appears in the urban development. Static traffic is a form of traffic relative to the existence of dynamic traffic, the two interrelated and influence each other. For vehicles in a city, it is dynamic when traveling and static when parked[1]. With the increasing number of vehicles in the city, the demand for parking facilities is also increasing, if the two are out of balance, the parking facility is difficult to meet the growing demand for vehicles, which will lead to parking problems.

The intelligent parking ecosystem integrates all aspects of society: from the driver to the parking lot to the Internet of things terminals, even to airports, shopping centers, and universities[2]. Management and dissemination of real-time parking information can make both the government and drivers make informed travel decisions. Intelligent parking system integrate cutting-edge technology into a system, ranging from parking terminal sensors to smartphones APPs, from cloud computing to big data analysis[5], from Online To Offline services to mobile payments.

To solve this problem, the country support the development of intelligent parking system both from hardware configuration and software development. With the rapid development of terminals and wireless networks, the majority of drivers can access to parking lots information in real-time. By building a cloud platform and intelligent parking terminal system driven by big data[5], We can effectively integrate, analyze and forecast traffic conditions, parking data and user behavior, and recommend reasonable travel routes and parking plans for users, which will bring great convenience to users[4].

\section{SYSTEM DESIGN}

The intelligent parking system design based on the cloud platform, we developed a real-time parking query system on Android[3], which can locate the driver location, parking location query, path planning, registration, advance payment and other functions.

We use the application connected with the database to realize the data connection between mobile client and networks; establish relevant databases; Implement real-time data communication and other functions; Complete intelligent parking based on Android real-time query system.

According to the "parking assistant" APP information, the system framework can be divided into the following layers:

1. UI presentation layer: Android client interface and all related controls display.

2. Application layer: Parking query, positioning, path planning, charging and other related functions.

3. Data layer: local database design and implementation, background database connectivity and real-time data updates.

The specific system architecture shown in Figure 1.

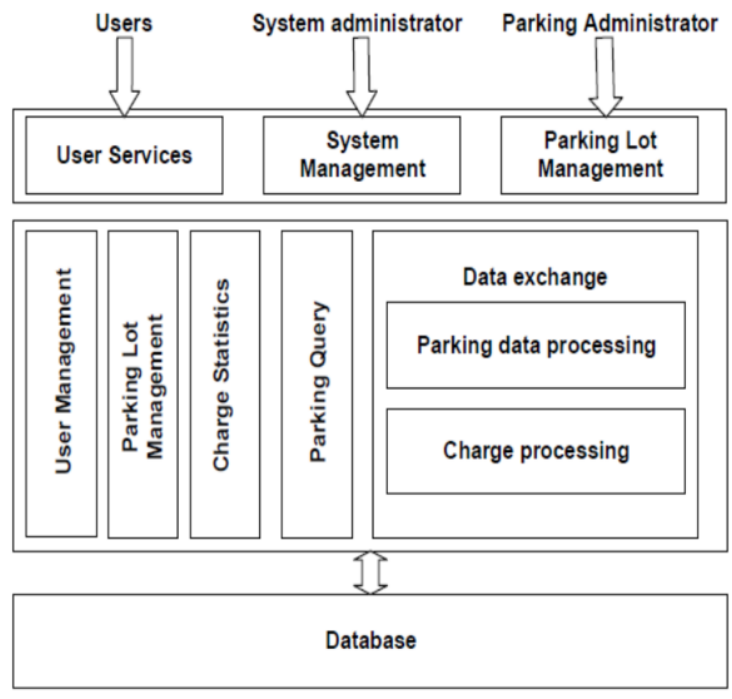

Figure 1. System framework

Parking and parking-related data updates through the TCP protocol. Users can use the system according to the UI presentation layer, including register, login, query, parking reservations and other modules. The administrator is responsible for system maintenance, database update 
management, user information management and charge management.

\section{INTELLIGENT PARKING SYSTEM UNDER CLOUD PLATFORM}

SQLite is adopted in the system design. It is a relational database management system conforming to ACID. It is contained in a relatively small $\mathrm{C}$ library and take up very little resources. In the embedded device, only a few kilobytes of memory is needed. In order to realize the function of parking pre-selection and parking in the system, the system can obtain the location of the parking lot in real time by using the mobile terminal positioning technology and network positioning technology of Baidu map.

The system is divided into pre-selected parking module, parking information module, driving route module and parking charging module, so that it can meet the different needs of users on the basis of cloud platform.

In the pre-selected parking module, mainly contains the current coordinate positioning, the surrounding parking lot search, parking location labeling. The client needs to use the commercial electronic map. Firstly, Android GPS device can get the positioning information, but the location information mainly composed of longitude and latitude, which is not useful to ordinary users; Secondly, Android can use the map API, such as Google Map, Baidu Maps, and AMap. However, Google Map is very inconvenient to use in China, Baidu map has more point of interest (POI) information, positioning more accurate, faster search speed, so the system uses the Baidu map software development kit (SDK) to build this function module.

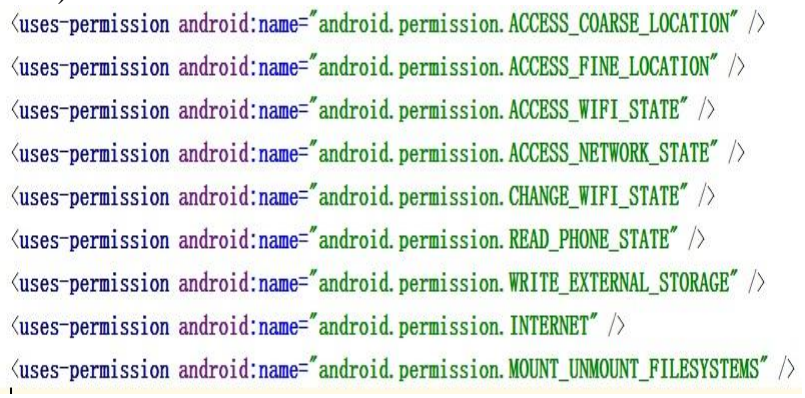

Figure 2. Baidu map authority

With the map API interface, the system can access Baidu map services and data, including geocoding capabilities. Geocoding provides the ability to convert between geographic coordinates and addresses. Forward geocoding can translate a Chinese address or the description of place into a corresponding location on the surface of the Earth; reverse geocoding can convert address coordinates of the Earth's surface into standard address. According to the latitude and longitude of current location provided by users, the server feeds back the parking lot near the user. Then the user query the specific parking lot details through the client. According to the selection of users, the server feeds back the parking lot details to the client. The information of the vacant parking space in the parking lot is real-time, so the real-time update requires the system to poll the parking management system server at regular intervals to confirm that the vacant parking information of the system client is consistent with the actual vacancy information of the parking lot. The API of Baidu map requires a certain authority to use, the specific call process shown in Figure 2:

The parameters such as radius and keywords are needed when the user search the surrounding parking lot. The Baidu Map SDK provides three types of POI searches: Peripheral, Regional, and Intra-city. The system uses a peripheral search type. First, the system needs to create a search POI instance named mPoiSearch, and then set the relevant parameters, the definition of POI parameters is shown in Figure 3.

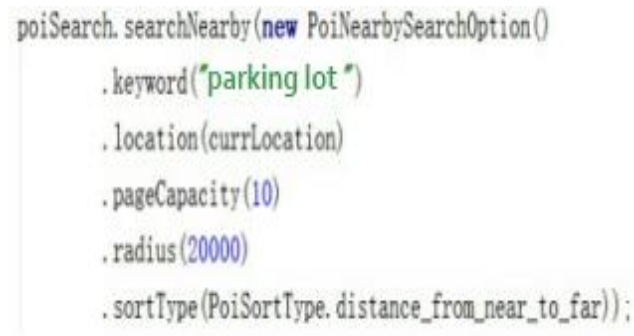

Figure 3. Definition of POI search parameter

In the driving route module, path planning is carried out between the current position and the destination by acquiring the positioning information of the current vehicle position.

The general steps for path planning are as follows:

Environment modeling: Environment modeling is an important part of path planning. The purpose is to build an environment model which is easy to be used by computer in path planning. it abstracts the actual physical space into the abstract space that the algorithm can deal with and realizes the mapping between them.

Path search: The path search phase is based on the environment model and applies a corresponding algorithm to find a walking path, so that the predetermined performance function can obtain the optimal value.

Path smoothing: The path searched by the corresponding algorithm is not necessarily a feasible path that a moving body can walk. It needs to be further processed and smoothed to make it become a feasible path.

The system uses the latitude and longitude coordinates of the user's current position as the starting point, and the latitude and longitude coordinates of the parking lot selected by the user as the end point to inquire the driving route. The core code to initialize the route plan is shown in Figure 4.

\section{$\mathbb{m}$ RroutePlanSearch, drivingSearch ((new DrivingRoutePlanOption ()$)$ . from(mRoutLinePlanots. getStartPlanIode()) . to (mRoutLinePlanots, getTargetPlanlode()));}

Figure 4. Driving Route Planning

The intelligentization of the toll collection module makes the charging process intelligent, automatic and real 
time. It is used to query the information of users, parking lots, orders, etc., to improve the efficiency of charges, which can save expenses and time. The module includes user management, parking management, billing information, system maintenance, each unit implement add, delete, modify, query functions, which can handle related business. Through the intelligent real-time charging system, we can manage user behavior, implement real-time charge, which can regulate the charging mechanism, improve the phenomenon of low charging efficiency, poor customer experience, and standardize the existing charging process.

\section{PARKING APP DEPLOYMENT AND OPERATION}

Users are most concerned about the pre-selected parking module, the user enter into the APP from the desktop, when the network is available, the system automatically displays the current location and the nearby 10 parking lots, then marked with 1 to 10 out in accordance with the distance. The magnification level of the map is adapted to the screen size and contains all the parking lot search results. The main interface contains three menus: login, registration and reservation. After registration and login, the related items disappear, and you can view the user information after login. When the user clicks on the icon of the corresponding parking lot, there will be two buttons below, respectively, the name of the parking lot button, you can jump to the parking detail page; another "to this" can jump directly to the driving route interface.

When the user clicks on the parking lot button, the user will jump to the page which display the parking lot name, address, total parking spaces and free parking spaces, and can display the parking unit price and parking forecast. Click "Go there" button to jump to the driving directions page; Click the top right "I'm here" button to jump to the parking cost estimation interface. As shown in Figure 5. The driving route map is drawn on the driving route page, the path planning prompt is shown at the bottom, and the "I'm there" button at the top right can jump to the parking cost estimation interface. According to the parking lot information displayed on Baidu map, the user can select the reservation function in the main interface, and enter the parking lot name, start parking time and end parking time to reserve the parking space. The system calculates the total parking fee based on the parking unit price, and displays the name of the parking lot selected by the user and license plate number of the user. As shown in Figure 6. After confirming the reservation information, the user can pay the parking fee in advance by the parking cost estimation function, which brings great convenience to the user, as shown in Figure 7.

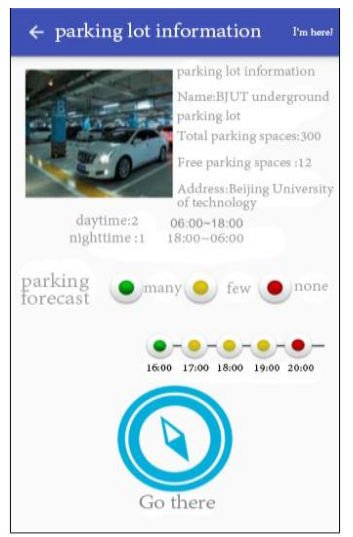

Figure 5. Parking lot information

Payment

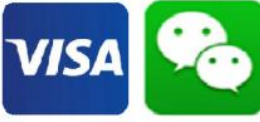

select payment mode

Figure 6. cost estimation

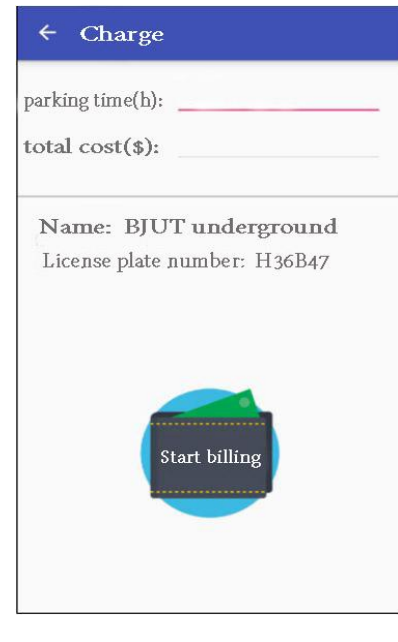

Figure 7. Payment module

\section{SYSTEM PERFORMANCE ANALYSIS}

The system performance can be test by running Power Tutor, WeTest and WiMAT benchmarks in the real machine environment. To provide a variety of software evaluation can have a comprehensive understanding of the system performance. The following is the test items, the performance comparison shown in Table 1. 
TABLE I. PERFORMANCE COMPARISON

\begin{tabular}{ccccccc}
\hline Seral number & APP name & CPU usage & Memory usage MB & $\begin{array}{c}\text { Power consumption } \\
\mathrm{J} / \mathrm{min}\end{array}$ & $\begin{array}{c}\text { Network traffic } \\
\mathrm{MB} / \mathrm{min}\end{array}$ & Package size MB \\
\hline 1 & Our System & $4 \%$ & 10.13 & 0.165 & 0.21 & 9.37 \\
2 & P+ parking & $7 \%$ & 18.01 & 3.313 & 0.45 & 1.28 \\
3 & Worry-free & $8 \%$ & 20.10 & 0.209 & 0.14 & 8.85 \\
4 & ETCP & $6 \%$ & 10.25 & 0.472 & 0.54 & \\
\hline
\end{tabular}

1.Power consumption: the consumption in the general use of scenes and state;

2.CPU usage: the consumption of network traffic in the general use of scenes and state;

3.Memory consumption: the memory usage in the general use of scenes and state;

Installation package size: the APP package size (without data files);

From the data analysis, Our system has obvious advantages compared with other systems, in addition to parking pre-selection, path planning, parking fees and other function which is for the convenience of users, the performance is also significantly better than other systems.

\section{CONCLUSION}

In this paper, we study from the domestic and international outstanding intelligent parking system, including the Parking in United States, the Parking Right UK and worry-free parking and $\mathrm{P}+$ parking China, and analyze the user demand for intelligent parking systems. Combined with the technical of Android platform, we design and implement the intelligent parking platform based on real-time query system. By registering and logging in, the user can view the surrounding parking lots information in real time, reserve vacant spaces and reach the destination under the driving route planning provided by system, which can alleviate the traffic congestion caused by the parking problem to a certain extent, and can effectively improve the utilization rate of the parking lot, which can avoid the inefficiency of owners looking for parking spaces which results in wasted time and expense.

This research was supported by the Beijing Municipal Science and Technology Project (Grant No. Z151100002615032), along with other government sponsors. The authors would like to thank the reviewers for their efforts and for providing helpful suggestions that have led to several important improvements in our work. We would also like to thank all the teachers and students in our laboratory for helpful discussions.

\section{REFERENCES}

[1] Xi, Min, et al. Application of Internet of Things in Intelligent Parking Management System. Computer Knowledge and Technology 7,27-44 (2011)

[2] Liu, Hua, et al. Design and Application of Decision Support System for Bulky transportation. Journal of Highway and Transportation Research and Development 2,3-8 (2015)

[3] Shi, Guang, Wei, Weihua, Wei, Jinwen. and R. I. Davis. Remote location system design based on Android 4.4 and Baidu map Application of MCU and Embedded System 11,39-41 (2014)

[4] Zhang, Yuexia, Liu, Yongchao, Wang, Jiaqing, et al. Design of intelligent parking lot based on android and embedded system. Computer Measurement and Control 23, 2830-2833 (2015)

[5] Li, Binya, Wu, Yunping. Intelligent Parking Software Based on Big Data. Electronic Measurement Technology 38, 44-47 (2015) 